\title{
Interpretation of the pinched point position in human skin memristor measurements
}

\author{
O. Pabst ${ }^{1}, \varnothing . G$. Martinsen ${ }^{1,2}$ \\ ${ }^{1}$ Department of Physics, University of Oslo, Oslo, Norway \\ ${ }^{2}$ Department of Clinical and Biomedical Engineering Oslo University hospital, Oslo, Norway
}

\begin{abstract}
It is shown that human skin acts like a memristor but systematic studies on skin memristance are not published, yet. If e.g., a low frequency voltage is applied to human skin and the current is measured, a hysteresis loop can be seen in the voltage-current plot. In skin measurements, the pinched point of the hysteresis loop can be shifted from the coordinate origin. In this paper, possible reasons for this observation shall be investigated and interpreted by the use of simulation. Two example measurements on human skin are presented, as well.
\end{abstract}

Keywords - Skin memristor, Bioimpedance, memristorcapacitance- parallel connection, non-linear properties.

\section{INTRODUCTION}

Non-linear properties can be observed in human skin if an electrical signal is applied [1, 2]. In [1] the observations are explained by electro-osmosis. In fact, it is shown that human skin acts like a memristor [3]. A model based on the observations in [1], is given. Human sweat contains ions. In theory, the electro-osmotic effect causes the ions to move, changing the level of sweat duct filling. Sweat ducts, that are highly filled, will conduct electrical current very well, while the resistance of less filled ducts is much higher. If e.g. a low frequency sinus voltage is applied to the skin, the filling of the ducts will change during a period, causing a change in the measured current. The resulting V-I plot will show a pinched hysteresis loop. A "memristor" (composition of "memory" and "resistor") is defined as the fourth electrical passive element and was first introduced in [4] and a pinched hysteresis loop is its "fingerprint" [5].

The V-I plots of skin memristor measurements can vary quite largely between measurements and subjects. In biological systems in general (like human skin), the position of the pinched point is shifted from the coordinate origin. [5].

In this paper, the influence of a DC offset to the applied voltage and a capacitance in parallel to the memristor shall be investigated as possible origins for the shift of the pinched point. First, two example measurements on human skin will be presented to show its properties. Second, simulations based on the Hewlett Packard (HP) - memristor [6] are done, in order to investigate the effect of both, an offset and a parallel capacitance, on the V-I-plot.

\section{MATERIALS AND METHODS}

\section{A. Example measurements}

The example measurements were done on two healthy test subjects (male and female, age: 29 and 24 years) in March and April 2017 at the University of Oslo. A custombuilt three-electrode measurement system that is based on the one presented in [7] is used for the recordings. A constant AC voltage with amplitude $2 \mathrm{~V}$ and frequency $0.1 \mathrm{~Hz}$ was applied to the skin for two periods and the corresponding current was measured. Signal generating and reading were done with 500 samples per period.

As current carrying electrode, a saline solution $(0.9 \%$ $\mathrm{NaCl}$ ) was used and the left hand was placed in it during the recording. The voltage was applied to the solution by several $\mathrm{Ag} / \mathrm{AgCl}$ electrodes. The reference ${ }^{1}$ electrode was placed in the center of the left forearm on the ventral side in $4 \mathrm{~cm}$ distance to the wrist. The same applied to the measuring electrode $^{2}$ with the difference that the distance to the wrist was $7 \mathrm{~cm}$. The recordings were started 5 minutes after the measuring electrode was placed.

\section{B. Simulation}

The memristor model used for the simulations in this paper is based on the HP - memristor, presented in [6]. Its memristance (in analogy to resistance) is dependent on an internal state ${ }^{3}$. The equations that describe the memristor [6] are given by

$$
\begin{aligned}
& M(x)=R_{o n} \cdot x+R_{o f f} \cdot(1-x) \\
& \frac{d x}{d t}=\frac{\mu_{v} \cdot R_{o n}}{D^{2}} \cdot \mathrm{I}_{\mathrm{M}}(\mathrm{t})
\end{aligned}
$$

\footnotetext{
${ }^{1}$ Type: Kendall $1050 \mathrm{NPSM}$ which is a pre-wired $\mathrm{Ag} / \mathrm{AgCl}$ electrode that is pre-gelled with a solid hydrogel (active area $5.05 \mathrm{~cm}^{2}$ ).

${ }^{2}$ Type: Pre-wired dry $\mathrm{Ag} / \mathrm{AgCl}$ electrode with an active area of $0.283 \mathrm{~cm}^{2}$ from the company Wuhan Greentek PTY LTD. Tape is used in order to attach the electrode to the skin.

${ }^{3}$ Determined by the expansion of a doped titanium dioxide region vs. an undoped one of the same material.
} 
with the normalized width $\mathrm{x}$ as the internal state ${ }^{4}$. In the used presentation ${ }^{5}$, can range from 0 to 1 and the initial state $\mathrm{x}_{0}$ describes the internal state at the beginning of the simulation. In order to consider saturation a window function [8] is used. The simulations in this paper were done by the use of Maple (Version 2016.2). The differential equations were solved numerically by the use of the Fehlberg fourth-fifth order Runge-Kutta method ${ }^{6}$. Several simulation results ${ }^{7}$ based on the same equations and algorithms are shown in [9]. Each simulation in this paper is done over two periods by the use of a constant sinus voltage $\left(\mathrm{V}_{\mathrm{S}}(\mathrm{t})\right)$ with amplitude $2 \mathrm{~V}$ and frequency $2.5 \mathrm{~Hz}$ as source. The current through the memristor can be calculated by

$$
\mathrm{I}_{\mathrm{M}}(\mathrm{t})=\frac{\mathrm{V}_{\mathrm{M}}(\mathrm{t})}{M(x)}
$$

with $\mathrm{V}_{\mathrm{M}}(\mathrm{t})$ as the voltage over the memristor.

In simulation 1, the influence of a DC offset on the V-I plot of a single memristor was investigated. Thus, a DC offset of $-0.1 \mathrm{~V}$ was added to the applied voltage. The resulting current was plotted against the sinus voltage without the DC offset (see IV Discussion for further explanation).

In a second simulation, the influence of a capacitance that is connected in parallel on the V-I-plot was investigated. The overall current in the memristor-capacitance-parallel circuit is described by

$$
\mathrm{I}_{\text {para }}(\mathrm{t})=\mathrm{I}_{\mathrm{M}}(\mathrm{t})+\mathrm{I}_{\mathrm{C}}(\mathrm{t})=\frac{\mathrm{v}_{\mathrm{S}}(\mathrm{t})}{M(x)}+C \cdot \frac{\mathrm{dV}_{\mathrm{S}}(\mathrm{t})}{\mathrm{dt}}
$$

with a capacitance value of $300 \mathrm{nF}$ used in this paper. The simulation was done for two different initial states $\mathrm{x}_{0}(0.3$ and 0.4 ) of the memristor.

\section{RESULTS}

\section{A. Results from the example measurements}

The two example measurements recorded on human skin are shown in figure 1 . The pinched points of the hysteresis loops of both periods in figure 1 a) are located in the first quadrant of the V-I plot and close to the coordinate origin. The arrow indicates the position of the pinched point in the second period, which is similar to the one in the first period.

A small cumulative state change from period one to two

${ }^{4} R_{\text {on }}=0.1 \mathrm{k} \Omega, R_{\text {off }}=16 \mathrm{k} \Omega, \mu_{v}=10^{-14} \frac{\mathrm{m}^{2}}{\mathrm{sV}}, D=10^{-8} \mathrm{~m}$

${ }^{5}$ Equations (1), (2) are slightly adapted in this paper. Instead of the total length $\mathrm{w}$ of the doped region, the normalized (to one) length $\mathrm{x}$ is used.

${ }^{6}$ Initial numerical method in Maple if the command "dsolve" is used and the option "type=numeric" is chosen.

${ }^{7}$ E.g. memristance change over time for a single HP memristor if a constant AC voltage is applied. can be seen since the amount of the current is slightly smaller in the second period. In figure $1 \mathrm{~b}$ ) the pinched point of the hysteresis loop in the second period is located in the first quadrant like indicated by the arrow.
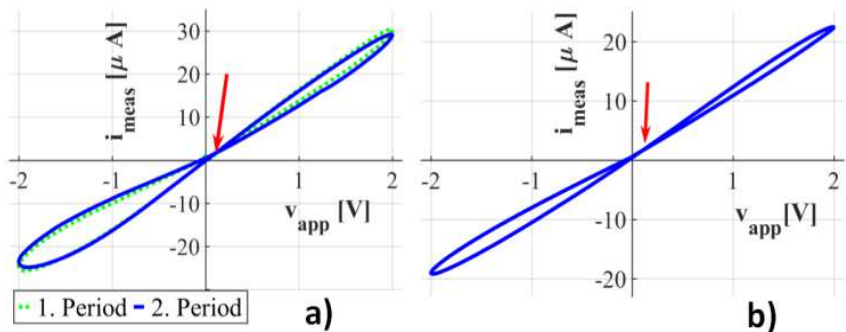

b)

Fig 1. Real measurements: V-I plots obtained from two example measurements on skin. The recordings were done on the left forearm over two periods. Applied sinus voltage: $\mathrm{f}=0.1 \mathrm{~Hz}$, amplitude $=2 \mathrm{~V}$, (and a DC level offset of 0 V). a) Subject 1, b) Subject 2, only second period.

\section{B. V-I plots obtained by simulation}

In figure 2, the results from simulation 1 (effect of a DC offset on the V-I plot) are shown. The position of the pinched point is the same in both periods and indicated by an arrow. The amount of the current is slightly smaller in the second period.

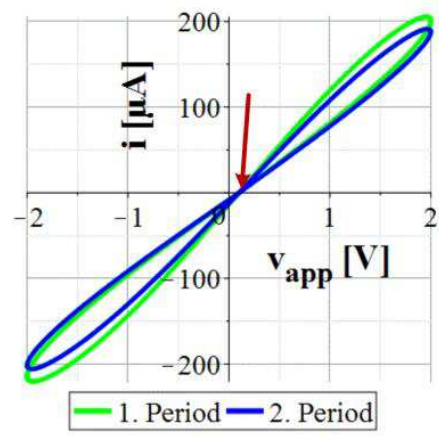

Fig 2. Simulation 1: Influence of a DC offset on the V-I-plot of a single HP memristor obtained by simulation over two periods. A constant sinus voltage with $\mathrm{f}=2.5 \mathrm{~Hz}$, amplitude $=2 \mathrm{~V}$, and a DC offset of $-0.1 \mathrm{~V}$ is used for the simulation. The scale on the voltage axis is treated as there would be a DC level offset of $0 \mathrm{~V}$. The initial state $\mathrm{x}_{0}$ was 0.3 .

The results of simulation 2 (parallel connection of a HP memristor and a capacitance) are presented in figure 3 . In the presented V-I plot the hysteresis curves for two different initial states of the HP memristor are shown. Each simulation run is done over two periods. No cumulative state change over periods occurred in this simulation and the curves from the first and second period of each run are 
overlapping. The two arrows indicate the different positions of the pinched points of both simulation runs.

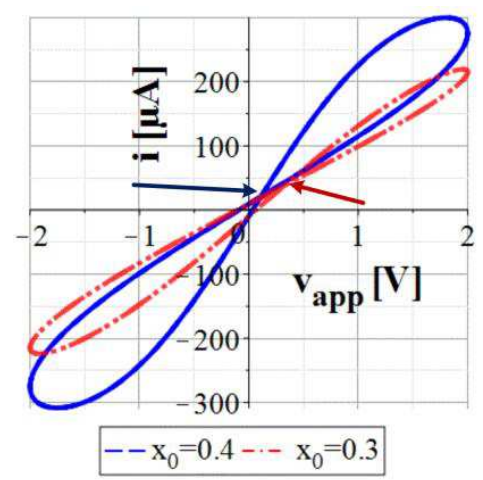

Fig 3. Simulation 2: V-I-plot of a HP memristor in parallel with a capacitor $(300 \mathrm{nF}$ ) obtained by simulation. The results of two different simulation runs are presented in the plot. A constant sinus voltage with $\mathrm{f}=2.5 \mathrm{~Hz}$, amplitude $=2 \mathrm{~V}$, (and a DC offset of $0 \mathrm{~V}$ ) is used for both. The initial state $\mathrm{x}_{0}$ of the HP memristor differed between the runs as it is shown in the legend. Each simulation run was done and is presented over two periods which cannot be seen since the results do not differ between periods.

\section{DISCUSSION}

\section{A. Difference between the skin memristor and the HP model}

The HP memristor model and the skin memristor are of course different. The hysteresis curve of the used HP memristor model is symmetric regarding the coordinate origin and does not change over periods if a constant sinus voltage without offset is applied ${ }^{8}$. This means that the internal state change happens with the same speed into both directions (decreasing and increasing). However, the state change of a skin memristor may happen unsymmetrically since it is likely that filling of the sweat glands occurs faster than emptying. As a consequence the cumulative state change over periods can probably also happen in absence of a DC potential. Another difference between the skin memristor and the HP memristor model is the range of the memristance. It is from $0.1 k \Omega$ to $16 k \Omega$ for the used HP memristor model. The memristance in a skin measurement depends on the used measuring electrode type and size. With the used setup, the lowest and highest values of the skin memristor are about 50 times larger than the ones of the HP memristor model (experiences from an unpublished systematic study). To obtain somehow comparable results to the skin measurement, initial states of high memristance $\left(\mathrm{x}_{0}=0.3\right.$ and $\left.\mathrm{x}_{0}=0.4\right)$ were used for the simulation. In order

\footnotetext{
${ }^{8}$ This statement is only valid as long as the HP memristor does not reach its maximum or minimum value during one period (see [9]).
}

to avoid states of very low memristance, a much higher signal frequency was used for the simulation $(\mathrm{f}=2.5 \mathrm{~Hz})$ than it was used for the measurement $(\mathrm{f}=0.1 \mathrm{~Hz})^{9}$. Still, the simulated current was about 10 times higher than the measured current.

However, independent of the chosen model ${ }^{10}$, the simulations provide general insights on how a DC offset and a capacitance in parallel will influence the V-I-plot and the position of the pinched point in the hysteresis curve. These influences will also affect skin measurements.

The two measurements that are presented in figure 1 are just examples to demonstrate the shift in the pinched point. Variances in terms of skin conductance and e.g. the maximum current and the area of the loop in the V-I plot can be expected among several test subjects and runs, which is not subject of this paper.

\section{B. Influence of a DC offset}

Endogenous potentials appear naturally on skin and vary among different skin sites. Potential differences between the reference electrode and the measuring electrode will add as a DC offset to the applied voltage. A possible half-cell potential caused by the use of different electrode types will add as an additional DC offset. While the latter one can be reduced by the electrode choice, it will be difficult to control the skin potential difference offset. In order to reduce its occurrence, skin sites of same potential can be used for the recording [10].

To investigate the effect of any DC offset, a voltage of $0.1 \mathrm{~V}$ was added to the sinus voltage source in simulation 1 . The value was chosen based on the example measurement that is presented in figure $1 \mathrm{a}$ ). In this case, the current in the second period became slightly smaller. Nevertheless, it is also observed in similar measurements that the amount of current increases over periods (unpublished results). If a DC offset of e.g. $+0.1 \mathrm{~V}$ is chosen for the simulation instead, the amount of current will increase over periods which is in accordance with the observations that were done before and the fact that the skin potential difference will add as a positive offset.

For the measurements in this paper, a constant voltage source is used and the current is measured. In the V-I-plot each measured current value is plotted against the corresponding voltage value which is given by the voltage source. Because of the DC offset that adds to the applied

\footnotetext{
${ }^{9}$ The higher the frequency the smaller the state change.

${ }^{10}$ In [3] a model of skin memristance is suggested but the HP memristor model was used for the simulation since it is much better known.
} 
voltage, the actual voltage over the skin is different. To consider this fact, two different voltages are used for simulation 1. One with an additional offset that is used for the simulation and one without the offset that is used to relate the calculated current values and plot them in the V-I-plot.

Each DC offset will shift the pinched point among the voltage axis. This shift is independent of the actual state of the memristor.

\section{Influence of a capacitance in parallel to a memristor}

In measurements on skin a capacitance will be in parallel to the skin memristor. The capacitance is mainly related to the humidity of the stratum corneum (in the low frequency range) [11] and values up to $100 \mathrm{nF}$ and above are realistic. The influence on the V-I plot of a capacitor in parallel depends on its capacitance value, the signal frequency and the internal state of the memristor. As long as the memristor is dominating in the parallel circuit, the position of pinched point is not much affected by the capacitance. The simulations (see figure 3 ) were done for two different initial states of the HP -memristor. With $\mathrm{x}_{0}=0.4$ the influence of the used capacitance can be already seen. The pinched point is shifted from the coordinate origin. The effect of the capacitance becomes even more obvious if $\mathrm{x}_{0}=0.3$ is used as initial condition. The memristance during this simulation run is higher compared to the run with $\mathrm{x}_{0}=0.4$. As the memristance becomes higher, the influence of the capacitance on the measurement increases. The pinched point is even more distant from the coordinate origin. Furthermore, it can be seen that the hysteresis curve becomes more and more unsymmetrical (because of the influence of the capacitance). Both simulation runs are done over two periods but since there is no DC offset, no cumulative state change can be observed.

In a measurement on skin in which the memristance can change cumulatively over periods, the influence of the capacitance differs. Thus, it will be possible that the pinched point moves during the recording over several periods.

\section{v. Conclusion}

In this paper, the results from two example experiments on skin are shown. The pinched point of the hysteresis curve is slightly shifted from the coordinate origin. By simulation in which the HP memristor model is used, it can be shown that both, a DC offset and a capacitance that is connected in parallel, will influence the position of the pinched point in the V-I-plot. Furthermore, any DC offset will cause a cumulative state change of a memristor over several periods. For skin memristor measurements, both a capacitance in parallel and a DC offset will contribute naturally to the measurement.

\section{ACKNOWLEDGMENTS}

This work has been performed within DIATECH@UiO, a strategic research initiative of the Faculty of Mathematics and Natural Sciences, University of Oslo.

\section{Conflict of InTEREST}

The authors declare that they have no conflict of interest.

\section{REFERENCES}

1. Grimnes, S. (1983) Skin impedance and electro-osmosis in the human epidermis. Medical and Biological Engineering and Computing, 21(6), 739-749. doi: 10.1007/BF02464037

2. Yamamoto, T., \& Yamamoto, Y. (1981) Non-linear electrical properties of skin in the low frequency range. Medical and Biological Engineering and Computing, 19(3), 302-310. doi: $10.1007 / \mathrm{BF} 02442549$

3. G. K. Johnsen, C. A. Lütken, Ø. G. Martinsen, and S. Grimnes (2011) Memristive model of electro-osmosis in skin, Physical Review E, vol. 83, no. 3, p. 031916. doi: https://doi.org/10.1103/PhysRevE.83.031916

4. L. Chua, (1971) Memristor-the missing circuit element, Circuit Theory, IEEE Transactions on, vol. 18, no. 5, pp. 507-519. doi: 10.1109/TCT.1971.1083337

5. L. Chua (2014) If it's pinched it's a memristor, in Memristors and Memristive Systems, pp. 17-90, Springer.

6. Strukov DB, Snider GS, Stewart DR, Williams RS. The missing memristor found. Nature. 2008;453(7191):80-83. doi: http://dx.doi.org/10.1038/nature06932.

7. Grimnes S (1983) Impedance measurement of individual skin surface electrodes. Med. Biol. Eng. Comput., 21, 750-755. doi: 10.1007/BF02464038

8. Joglekar YN, Wolf SJ. The elusive memristor: properties of basic electrical circuits. European Journal of Physics. 2009;30:661 doi: http://dx.doi.org/10.1088/0143-0807/30/4/001.

9. Pabst, O., \& Schmidt, T. (2013). Frequency dependent rectifier memristor bridge used as a programmable synaptic membrane voltage generator. Journal of Electrical Bioimpedance, 4(1), 23 32. doi: http://dx.doi.org/10.5617/jeb.539

10. Martinsen, Ø. G., Pabst, O., Tronstad, C., \& Grimnes, S. (2015). Sources of error in AC measurement of skin conductance. Journal of Electrical Bioimpedance, 6(1), 49-53. doi: $10.5617 /$ jeb.2640

11. Martinsen, Ø. G., Grimnes, S., \& Karlsen, J. (1995). Electrical methods for skin moisture assessment. Skin Pharmacology and Physiology, 8(5), 237-245. doi: 10.1159/000211353

$\begin{array}{ll}\text { Author: } & \text { Oliver Pabst } \\ \text { Institute: } & \text { Department of Physics, University of Oslo } \\ \text { Street: } & \text { Sem Sælands vei } 24 \\ \text { City: } & \text { Oslo } \\ \text { Country: } & \text { Norway } \\ \text { Email: } & \text { oliverpa@ mail.uio.no }\end{array}$

